\title{
Audit of clinical-laboratory practices in haematology and blood transfusion at Muhimbili National Hospital in Tanzania
}

\author{
ABEL N. MAKUBI ${ }^{*}$, COLLINS MEDA², ALEX MAGESA ${ }^{2}$, PETER MINJA2, JULIANA MLALASI ${ }^{2}$, \\ ZUBEDA SALUM ${ }^{2}$, RUMISHA E. KWEKA², JAMES RWEHABURA², AMRANA QUARESH ${ }^{3}$, PIUS M. \\ MAGESA $^{1}$, DAVID ROBERT ${ }^{4}$, JULIE MAKANI ${ }^{1}$ and EPHATA KAAYA ${ }^{1}$ \\ ${ }^{1}$ Muhimbili University of Health and Allied Sciences, School of Medicine, P.O. Box 65001, Dar es Salam, Tanzania \\ ${ }^{2}$ Muhimbili National Hospital, Dar es Salaam, Tanzania \\ ${ }^{3}$ Deparment of Paediatrics, Great Omond Hospital, London, United Kingdom \\ ${ }^{4}$ National Blood Transfusion Service, Oxford University, London, United Kingdom
}

\begin{abstract}
In Tanzania, there is paucity of data for monitoring laboratory medicine including haematology. This therefore calls for audits of practices in haematology and blood transfusion in order to provide appraise practice and devise strategies that would result in improved quality of health care services. This descriptive cross-sectional study which audited laboratory practice in haematology and blood transfusion at Muhimbili National Hospital (MNH) aimed at assessing the pre-analytical stage of laboratory investigations including laboratory request forms and handling specimen processing in the haematology laboratory and assessing the chain from donor selection, blood component processing to administration of blood during transfusion. A national standard checklist was used to audit the laboratory request forms (LRF), phlebotomists' practices on handling and assessing the from donor selection to administration of blood during transfusion. Both interview and observations were used. A total of 195 LRF were audited and $100 \%$ of had incomplete information such as patients' identification numbers, time sample ordered, reason for request, summary of clinical assessment and differential diagnoses. The labelling of specimens was poorly done by phlebotomists/clinicians in $82 \%$ of the specimens. Also 65\% (132/202) of the blood samples delivered in the haematology laboratory did not contain the recommended volume of blood. There was no laboratory request form specific for ordering blood and there were no guidelines for indication of blood transfusion in the wards/clinics. The blood transfusion laboratory section was not participating in external quality assessment and the hospital transfusion committee was not in operation. It is recommended that a referral hospital like $\mathrm{MNH}$ should have a transfusion committee to provide an active forum to facilitate communication between those involved with transfusion, monitor, coordinate and audit blood transfusion practices as per national guidelines.
\end{abstract}

Keywords: audit, laboratory, haematology, practices, hospital, Tanzania

\section{Introduction}

Audit is defined as a quality improvement process that seeks to improve patient care and outcomes through systematic review of care against explicit criteria and the implementation of change (Scrivener et al., 2002 ). It is a part of continuous quality improvement process and a key element of clinical governance. Laboratory-based audits evaluate components of laboratory services; providing feedback to staff and users about the

* Correspondence: Abel Makubi; E-mail: Makubi55@gmail.com 
laboratory functions and efficiency. There are three components involved in laboratory auditing namely: pre-analytical phase, analytical phase and post-analytical phase (Erasmus \& Zemlin, 2009). Due to the laboratory quality cycle, reliability cannot be achieved in a clinical laboratory through the control of accuracy in a single component of testing process alone. There should be a certification on the whole laboratory functions, but not on single analytical process (Wiwanitkit, 2001). Under the broad umbrella of the pre-analytical phase, the following are included: requesting test, specimen collection, handling and processing before complete distribution of test samples to multiple work stations (Erasmus \& Zemlin, 2009; Scrivener et al., 2002).

Studies indicate that mistakes in the pre-analytical phase alone can account to more than $50 \%$ of the mistakes in the testing cycle (Boone et al., 1995). In a study conducted in Italy, $6.6 \%$ of 189 pre-analytical phase and post-analytical phase mistakes were associated with inappropriate therapy (such as inappropriate transfusion or inappropriate heparin use) and in another 19\%, the mistakes were associated with inappropriate further investigations, thus increasing costs of care (Plebani \& Carraro, 1997). Very few studies have examined the frequency and impact of incomplete laboratory request forms. Laboratory errors are of utmost importance, as laboratory data influence $70 \%$ of medical diagnoses and can impact significantly on the success and cost of patient treatment. For these reasons previous efforts to reduce errors in the analytical phase have been abandoned (Bonini et al., 2002) since currently, pre- and post-analytical processes in the laboratory are more vulnerable to errors (Plebani, 2006) and sometimes the pre-analytical phase accounting to up to $68 \%$ of laboratory errors. This phase includes procedures which are not under the control of laboratory personnel and are mostly performed outside the laboratory. The procedures include completion of laboratory request forms, specimen identification, phlebotomy, sample handling and transportation to the laboratory (Vanker et al., 2010). Laboratory request forms provide information about the laboratory test requested and contain demographic data such as name(s), date of birth, subject's address, age, and sex. Other details include the ward, laboratory number, doctor's name, signature of the doctor, telephone or fax number of the doctor; clinical details, fasting status of the subject and the date of request.

There is no published data to describe internal auditing in blood transfusion and laboratory haematology practices in Tanzania. Auditing of current practice is a priority activity in haematology and blood transfusion as this would result in improvement of the quality of care and health services. At the Muhimbili National Hospital (MNH), laboratory request forms are filled by doctors and presented to the laboratory. During blood collection, after identifying the patient through the laboratory request form, phlebotomists are responsible for labelling the sample container and adding blood as recommended for a particular test. In this paper, we report an audit of laboratory practice in haematology and blood transfusion. First we evaluated the completeness of laboratory request forms (LRF) as checked by clinicians. Secondly, we assessed the laboratory practice in processing specimens in the haematology laboratory by phlebotomists and lastly we assessed the process chain from donor selection to administration of blood during transfusion. 


\section{Methods and Materials}

\section{Study area}

The study was conducted in November 2010 in the Haematology and Blood Transfusion section within the Central Pathology Laboratory (CPL) at MNH in Dar es Salaam, Tanzania. CPL is the largest laboratory in Tanzania performing various pathology laboratory tests including haematology and blood transfusion. $\mathrm{MNH}$ is a national referral hospital for patient from regional and other referral hospitals across the country. The Haematology and Blood Transfusion Laboratory receives approximately 250 specimens per day from within and outside MNH.

\section{Study design, Specimen and Sample size}

This was a descriptive cross-sectional study that was aimed at assessing the haematology and blood Transfusion practices in the Hospital. Specimens accompanied by filled request laboratory forms were received in the laboratory reception room for registration before being processed and report sent back to the ordering clinicians/wards/clinics. The audit involved specimens from patients who were seen on the day of the study. A total of 195 laboratory request forms and 202 blood specimens were randomly audited.

\section{Data collection}

Using a national standard checklist, filled LRF and containers with specimen from the patients were audited. Laboratory forms from clinicians were also audited to see if they were completed correctly and contained the required information. Audit of practice of phlebotomists in processing specimens was done by examining if specimens were correctly and completely labelled for the required information and contained the required volume of blood.

Audits in blood transfusion involved evaluating activities from donor selection to administration of blood during transfusion. The head of blood donation section was interviewed to evaluate the procedures in handling blood donors as well as processing blood, enquiring whether there were written policies for the procedures, whether there were procedure for issuing blood from the hospital blood bank and enquiring about the overall organization and management of hospital blood transfusion services. Some of the activities were cross checked with observation.

\section{Data analysis}

Data analysis was done using SPSS version 13.0 computer programme. Through descriptive statistics of frequencies, categorical variables were expressed in percentages. Manual marking for yes or no response in checklist was done for interview and observational variables.

\section{Ethical consideration}

Ethical approval for this study was sought from Muhimbili University of Health and Allied Sciences' Ethical Review Board. Permission to access the laboratory documents and specimens was obtained from the MNH authority and the heads of the sections in the Central Pathology Laboratory. 


\section{Results}

\section{Audit of laboratory request practices by the clinicians}

A total of 195 filled LRF from clinicians were audited to see if required information was completed. The results showed that patient's surname $(100 \%)$, patient's first name $(99 \%)$, date of birth or age (93\%), patients' identification numbers $(97 \%)$ and patient's gender $(99 \%)$, were adequately completed by the ordering clinicians (Table 1). However date and time the results are required $(2.6 \%)$ (Table 1$)$, the time sample was collected $(2 \%)$, time sample was ordered (3\%), (Figure1). In fact $100 \%$ of the LRF audited were incompletely checked for all information as required (Table 1$)$.

Table 1: Proportion of completeness for the laboratory request forms as filled/checked by clinicians (N=195)

\begin{tabular}{lll}
\hline Variable & Number & Percentage \\
\hline Patient's surname & 195 & 100 \\
Patient's first name & 193 & 99 \\
Date of birth /age & 182 & 93 \\
Gender of patient & 194 & 99.5 \\
Patient identification number & 189 & 96.9 \\
Location of patient at time of request & 169 & 86.7 \\
Date and time results are required & 5 & 2.6 \\
Summary of symptoms and signs & 144 & 73.8 \\
Patient's diagnosis & 74 & 37.9 \\
Reason for request & 58 & 29.7 \\
Name of the ordering person & 173 & 88.7 \\
Signature of the ordering person & 15 & 7.7 \\
Urgency for results & 56 & 28.7 \\
Date sample arrives in the lab & 63 & 32.3 \\
Time sample arrived in the lab & 50 & 25.6 \\
Completely labelled in all parameters & 36 & 17.8 \\
\hline
\end{tabular}

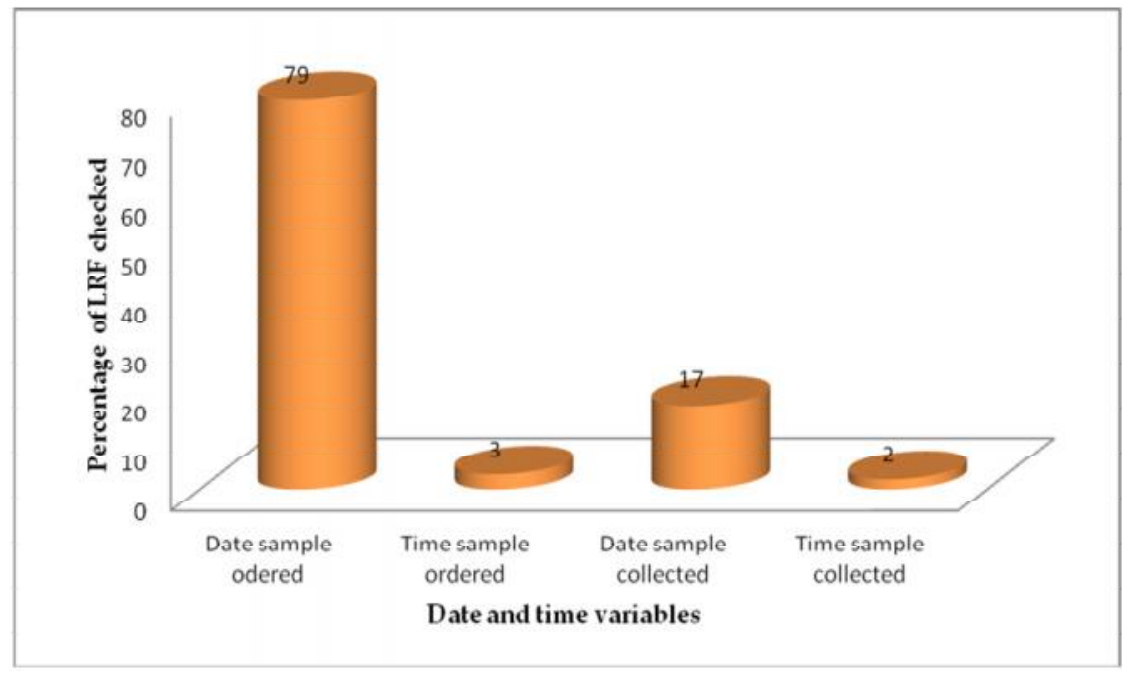

Figure 1: Proportion of 195 LRF checked for dates and times during test ordering and sample collection 


\section{Audit of phlebotomists' practices on handling specimens}

A total of 202 specimen containers were audited in the haematology laboratory and a total of 11 variables were examined to see whether the phlebotomists labelled the containers as required. This aspect revealed that labelling of specimens was poorly done by phlebotomists/clinicians as $82.2 \%$ of the specimens were incompletely labelled for the required parameters (Table 2). This includes inadequate labelling of signature of the sample collector $(10 \%)$, time the specimen was collected $(12 \%)$, patient's date of birth or age $(22 \%)$, and location to where results should be channelled (52\%). In addition 132 (65\%) of the blood samples delivered to the haematology laboratory did not contain the recommended volume of blood and $1.5 \%$ had already leaked (Table 2).

\section{Audits in Blood Transfusion (from donor to patient)}

The blood donation was practiced in line with the national guidelines except for the absence of physician at the post-donation section as well as absence of mechanisms for tracing donors. All blood units were screened for transmissible transfusion infections (TTIs) and there were standard operation procedure for screening, blood component separation, TTIs, and basic antibody screening. Whole blood, frozen plasma, red blood cells, and platelets were the only blood component prepared. There was no extended for extended antibody screening and identification (other than $\mathrm{ABO}$ and Rh-D).

Table 2: Proportion of blood samples checked for various parameters by phlebotomists as delivered to the haematology laboratory $(\mathrm{N}=202)$

\begin{tabular}{lll}
\hline Variable observed & Number & Percentage \\
\hline Patient's first name checked in container & 201 & 99 \\
Patient's surname checked in container & 201 & 99 \\
Patient's identification number checked in container & 143 & 70 \\
Patient date of birth or age checked in container & 46 & 22 \\
Location of patient at time of request & 106 & 52 \\
Date sample collected & 83 & 40 \\
Time sample collected & 25 & 12 \\
Signature of the sample collector & 21 & 10 \\
Blood collected in the right container & 201 & 99 \\
Blood filed to the required volume in the tube & 132 & 65 \\
Any leakage of the sample & 3 & 1.5 \\
\hline
\end{tabular}

The procedures ordering, issuing blood and its administration appeared to partly adhere to the current guidelines. This included proper documentation of the type and amount of blood required, documenting all units issued to the patients, using identification tag and checking/documenting for blood transfusion reactions However there were no specific laboratory request forms for ordering blood and no guidelines for indication for blood transfusion in the wards and clinics, especially with neonates and obstetrics.

The audit also found that the blood transfusion laboratory section was not participating in external quality assessment and the hospital transfusion committee was not functional. There were also no refresher courses on blood transfusion given to laboratory staff, nurses or 
clinicians.

\section{Discussion}

The healthcare system is increasingly dependent on reliable clinical laboratory services, which requires planned regular audits of laboratory operations, matched to the laboratory workflow phases. In Tanzania laboratory audits are done infrequently and at MNH few audits of the preanalytic phase have been carried out. In the pre-analytic phase of this audit the level of completion of laboratory request forms as completed by physicians was low in most of the variables with exception of patient's surname, patient's first name, date of birth or age, gender of patient and the test requested. This is comparable to similar studies done by Burton \& Stephenson (2001) and Olayemi \& Asiamah-Broni (2011) in which only the patient's full name was stated on the request forms they evaluated. This was not surprising since it was very likely that the request would have been turned down if the required test was not stated and the client's name was absent. Other variables such as patients' identification numbers, the time sample was ordered, reason for request of the test, summary of clinical assessment and differential diagnoses, were poorly documented by the clinicians.

The patient's demographic data is relevant because it helps in specimen identification and proper interpretation of results. In instances where samples from different subjects have the same or similar names, information such as the location of the subject, age and gender are important in identifying and sorting out both the subject and samples. Also reference ranges for some tests like the haemoglobin concentration vary with age and gender. The location/ward of the patient enables results to be immediately communicated to the clinician.

There was no clinical detail provided in $27 \%$ of the request forms sampled. This was comparable to results obtained in a similar study conducted in Pakistan (Sharif et al., 2007) but higher than results obtained in the United States (Nakhleh \& Zarbo, 1996). It has been demonstrated that provision of adequate clinical information prevents inappropriate investigations (Burton \& Stephenson, 2001; Zemlin et al., 2009) and also helps in correctly interpreting the test results. Absence of clinical information or misleading information leads to extraneous and unnecessary additional tests, which has definite resource management and demand implications. Also where interpretative comments are made on laboratory results, inadequate clinical information may lead to misleading and potentially harmful comments (Plebani, 2006). The date on which the requests were made was found on $92 \%$ of all the request forms.

Only $1.5 \%$ of the request forms carried the time the sample was taken. The labelling of specimens was poorly done by phlebotomists as well as clinicians. Most of the blood samples delivered in the haematology laboratory did not contain the recommended volume. Too much or less volume of blood than the recommended one may lead to false results on most of the haematological tests such cell blood count and coagulation tests. For example, an insufficient amount of blood in a sodium citrate tube will produce a dilution problem if the specimen is tested for coagulation studies and excessive amount of Ethylene diamine tetraacetic acid (EDTA) over blood will produce shrinkage of erythrocytes (Turgeon, 1999).

Lack of a specific request form for blood transfusion may lead to inappropriate blood 
request and lack of informative summary relevant to blood transfusion and donation. Lack of guidelines for blood transfusion indications in the wards/clinics was a serious finding in this study. Guidelines for indication of different blood components are important to reduce the unnecessary use of scarce blood and blood product and minimize the costs to the hospital. This also reduces the risk of transfusion associated infections to patient who did not necessarily need blood.

The lack of routine indirect antiglobulin test (IAT) raises question on the identification of clinically significant IgG antibodies such as ant-D and anti-K in our set up. The hospital transfusion committee was also found not in operation. The ministry recommends that a referral hospital like Muhimbili should have a transfusion committee to provide an active forum to facilitate communication between those involved with transfusion, recommend or perform practice audits, monitor transfusion practice compared to institutional, national or international benchmarks, provide education to effect change in practice.

Though the haematology and blood transfusion laboratory units were performing adequate numbers of tests, there were significant deficiencies in the laboratory with regard to completeness of LRF and poor labelling of specimen by phlebotomists and clinicians as well as lack of guidelines for indication of blood transfusion in the wards and clinics. It is recommended that a referral hospital like $\mathrm{MNH}$ should have a transfusion committee to provide an active oversight, facilitate communication between those involved with transfusion, recommend or perform practice audits and monitor transfusion practice

\section{Acknowledgements}

We would like to acknowledge the support of all staff in the department of haematology and blood transfusion who participated in this audit. The study received financial assistance from the DelPHE -MUHAS project.

\section{Conflict of interest}

There was no conflict of interest

\section{References}

Bonini, P., Plebani, M., Ceriotti, F. \& Rubboli, F. (2002) Errors in laboratory medicine. Clinical Chemistry 48, 691-698.

Boone, D.J., Steindel, S.J. \& Herron, R. (1995) Transfusion medicine monitoring practices: a study of the College of American Pathologists/Centers for Disease Control and Prevention Outcomes Working Group. Archives of Pathology E Laboratory Medicine 119, 999-1006.

Burton, J.L. \& Stephenson, T.J. (2001) Are clinicians failing to supply adequate information when requesting a histopathological investigation?. Journal of Clinical Pathology 54, 806.

Erasmus, R.T. \& Zemlin, A.E. (2009) Clinical audit in the laboratory. Journal of Clinical Pathology 62, 593-597. 
Nakhleh, R.E. \& Zarbo, R.J. (1996) Surgical pathology specimen identification and accessioning: a College of American Pathologists Q-Probes study of 1004115 cases form 417 institutions. Archives of Pathology \& Laboratory Medicine 120, 227-233.

Olayemi, E. \& Asiamah-Broni, R. (2011) Evaluation of request forms submitted to the haematology laboratory in a Ghanaian tertiary hospital. Pan African Medical Journal 33, 233-240.

Plebani, M. (2006) Errors in clinical laboratories or errors in laboratory medicine? Clinical Chemistry and Laboratory Medicine 44: 750-759.

Plebani, M., \& Carraro, P. (1997) Mistakes in a stat laboratory: types and frequency. Clinical Chemistry 43: 1348-1351.

Scrivener, R., Morrell, C., Baker, R., Redsell, S., Shaw, E., Stevenson, K., Pink, D. \& Bromwich, N. (2002) Principles for best practice in clinical audit. International Journal for Quality in Health Care 15, 87-97.

Sharif, M.A., Mushtaq, S., Mamoon, N., Jamal, S. \& Luqman, M. (2007) Clinician's responsibility in Pre-Analytical Quality Assurance of Histopathology. Pakistan Journal of Medical Science 23, 720-723.

Turgeon, M. L. (1999) Theory and Procedures: Clinical Haematology 3rd edition, page 22.

Vanker, N., Wyk, J.V., Zemlin, A.E. \& Erasmus, R.T. (2010) A Six sigma approach to the rate and clinical effect of registration errors in a laboratory. Journal of Clinical Pathology 63, 434-437.

Wiwanitkit, V. (2001) Types and frequency of preanalytical mistakes in the first Thai ISO 9002:1994 certified clinical laboratory, a 6 - month monitoring. BMC Clinical Pathology 1:5.

Zemlin, A.E., Nutt, L., Burgess, C.J., Eiman, F. \& Erasmus, R.T. (2009) Potential for medical error: incorrectly completed request forms for thyroid function tests limit pathologist's advice to clinicians. South African Medical Journal 99, 668-671. 